\title{
Aula invertida como herramienta para el logro de aprendizaje autónomo en estudiantes universitarios
}

\section{Flipped classroom as a tool for the achievement of autonomous learning in university students}

\author{
Danny Nicke Ventosilla Sosa \\ Ministerio de Educación del Perú, Perú \\ ORCID: https://orcid.org/0000-0002-4740-7583 \\ Héctor Raúl Santa María Relaiza \\ Universidad César Vallejo, Perú \\ ORCID: https://orcid.org/0000-0002-4546-3995 \\ Felipe Ostos De La Cruz \\ Universidad César Vallejo, Perú \\ ORCID: https://orcid.org/0000-0002-2718-3957 \\ Ana María Flores Tito \\ Universidad César Vallejo, Perú \\ ORCID: https://orcid.org/0000-0001-5769-1488
}

Received 10-12-20 Revised 11-25-20

Accepted 02-13-21 On line 02-27-21

*Correspondence

Email: elsanta17@gmail.com
Cite as:

Ventosilla, D., Santa María, H., Ostos, F., \& Flores, A. (2021). Aula invertida como herramienta para el logro de aprendizaje autónomo en estudiantes universitarios. Propósitos y Representaciones, 9(1), e1043. Doi: http://dx.doi.org/10.20511/pyr2021.v9n1.1043

(c) Universidad San Ignacio de Loyola, Vicerrectorado de Investigación, 2021.

(cc) EY-NC-ND This article is distributed under license CC BY-NC-ND 4.0 International (http://creativecommons.org/licenses/by-nc-nd/4.0/) 


\section{Resumen}

La investigación tuvo como objetivo determinar la influencia del aula invertida como herramienta para el logro de aprendizaje autónomo en estudiantes universitarios. El tipo de estudio fue aplicada y el diseño experimental de sub tipo cuasi experimental. La muestra estuvo conformada por 73 estudiantes distribuidos en 2 grupos de los cuales uno fue el grupo control (37) y el otro el grupo experimental (36), estudiantes de ambos sexos, para lo cual se utilizó un muestreo No Probabilístico de tipo intencional. Con relación a la técnica, ésta fue la encuesta como instrumentos el cuestionario que fue aplicado de manera virtual el cual pasó por dos fases antes de ser aplicado a la muestra de estudio (validez y confiabilidad), con relación a la confiabilidad se obtuvo un valor de 0,864 según KR20. Los resultados del pretest en relación con el aprendizaje autónomo un 37,6\% y 41,2\% de los estudiantes del grupo control (GC) y grupo experimental (GE) mantienen un nivel bajo, asimismo, un $57,1 \%$ y $41,5 \%$ presentan un nivel medio, por su parte, el 5,3\% del grupo control y el 17,3\% del grupo experimental se situó en el nivel alto. Se llegó a la conclusión que el aula invertida, permite al estudiante aprender de manera autónoma incorporando las TIC como elemento innovador en la trasformación de la adquisición de los nuevos aprendizajes de manera responsable.

Palabras claves: Aula invertida, Aprendizaje autónomo, Metodología.

\section{Summary}

The research aimed to determine the influence of the flipped classroom as a tool for the achievement of autonomous learning in university students. The type of study that was used was applied and the experimental design of a quasi-experimental sub type. The sample consisted of 73 students distributed in 2 groups of which one was the control group (37) and the other the experimental group (36), students of both sexes, for which an intentional Non-Probabilistic sampling was used. In relation to the technique, this was the survey as instruments the questionnaire that was applied virtually which went through two phases before being applied to the study sample (validity and reliability), in relation to the reliability a value was obtained of 0.864 according to KR20. The pre-test results in relation to autonomous learning $38.9 \%$ and $43.2 \%$ of the students in the control group (CG) and experimental group (EG) maintain a low level, likewise, $58.3 \%$ and 43 , $2 \%$ present a medium level, for their part, $2.8 \%$ of the control group and $13.6 \%$ of the experimental group were at the high level. It was concluded that the flipped classroom allows the student to learn autonomously, incorporating TIC as an innovative element in the transformation of the acquisition of new learning in a responsible manner.

Keywords: Flipped classroom, Autonomous learning, Methodology.

\section{Introducción}

El mundo globalizado que conlleva a cambios repentinos en diferente contexto donde el desarrollo social, científico, técnico y económico obliga a los países a plantear políticas públicas, sobre todo políticas en educación. La educación tradicional impartida en las aulas universitarias recae en el docente quien debe escribir en la pizarra todo lo relacionado con el área o curso académico, el papel del estudiante se concentra en tomar notas de la clase y hacer las actividades; ya sea de manera individual o grupal. (Sierra y Mosquera, 2020)

En las aulas universitarias se evidencia un gran desinterés hacia el aprendizaje, en la que cualquier elemento o factor puede ser distractor que resulte como influencia para que el estudiante no se concentre. Pero además los estudiantes sienten que la educación sigue siendo tradicional, monótona y poco aburridas. Se sabe que la educación hoy en día se percibe como una nueva situación, es decir, la educación se encuentra en la era de la revolución tecnológica, donde la necesidad de una computadora y las telecomunicaciones puede cambiar el estilo de enseñanza 
aprendizaje.(Rodríguez y García 2006).

El mundo y las sociedades están cambiando y evolucionado, sobre todo en la forma de aprender, en este sentido, es necesario buscar estrategias y metodologías que logre una interacción entre los docentes, estudiantes y tecnología, teniendo en cuenta esta última como un factor clave y de gran apoyo para el estudiante en material de aprendizaje. En este sentido, docentes en muchas universidades están tratando de cambiar la enseñanza tradicional - enfocada en cumplir el avance a partir de un plan de estudios - por una enseñanza basados en las necesidades de los estudiantes. La metodología que ha despertado el interés en los estudiantes es el aula invertida como una metodología centrada en trasladar la instrucción directa al exterior del aula con el fin de dar aprovechamiento y la interacción entre uno a uno entre profesor del aula y estudiante (Bergmann y Sams, 2014 y Limón, Cantera y Salinas, 2017). El aula invertida es una metodología en la que la instrucción directa se hace efectiva y eficiente cuando se realiza de manera individual.

Con el creciente uso tecnológico en las últimas décadas se ha promovido nuevas metodologías, como la metodología tecnológica, comparadas a metodologías tradicionales se aprecia un cambio, es decir las metodologías que hacen uso de las tecnologías, permite al estudiante el desarrollo del pensamiento crítico, mejora en la comunicación tanto escrita como oral y sobre todo el desarrollo de habilidades, logrando así un incremento integral de los conocimientos. El uso de la metodología tecnológica permite mejorar la gestión del conocimiento del estudiantes, esto debido a los recursos y herramientas proporcionadas por los docentes en cada sesión de clase, lo cual ayuda al estudiante estar activo en su proceso de enseñanza aprendizaje. (Kong, 2014; McLaughlin et al., 2014).

Las metodologías que hacen uso de las tecnologías, apuntan a tener en cuenta los principios de la educación superior en el continente Europeo, en la que estipula no solo en lograr las competencias prácticas sino en la adquisición de conocimientos teóricos mediante actividades prácticas. No obstante, hay que hacer hincapié en la participación tanto del docente como del estudiante, el docente es la persona encargada de la elaboración de los diferentes recursos y herramientas puestas a disposición del estudiante para su respectiva revisión antes de dar inicio a la explicación de la clase, logrando la participación activa del estudiante y motivarlo a la búsqueda de información relacionada a la clase. (Arráez, Lorenzo, A., Gómez y Lorenzo, G., 2018).

La tecnología avanza y obliga a crear nuevas metodologías para el proceso enseñanza aprendizaje, es allí que se habla del aula invertida, metodología enfocada en combinar los dos momentos que intervienen en la educación tradicional: actividades propias de la clase y la segunda actividades fuera de la escuela (Merla y Yáñez, 2016 y Madrid, et al., 2018). En este sentido, el Aula Invertida permite un aprendizaje para el docente, donde el manejo de la tecnología puede ser dentro o fuera del aula, permitiendo así utilizar las Tics como herramienta para apoyar el aprendizaje de los estudiantes. (Zacarías, 2016). El aula invertida tiene como objetivo promover a los docentes de herramientas metodológicas para la transformación del proceso de enseñanza, el aula invertida permite un aprendizaje activo, donde el estudiantes se involucra directamente con el aprendizaje (Carignano, 2016).

El aula invertida o "Flipped Classroom", influye en el aprendizaje activo, logrando la autonomía del estudiantes, asimismo, permite que el estudiante "haga uso de recursos como videos para la comprensión de contenidos, resolución de ejercicios y trabajos grupales, como actividades ideales para aplicar la teoría y profundizar el contenido". (Wendorff, 2019 y Archbold, Nuñez y Padilla, 2019). Asimismo. El Aula Invertida, "Flipped Classroom" o "Inverted Classroom", es vista como una forma de enseñanza de manera semipresencial o mixta ("blended learning") esto debido 
a su forma presencial y otra virtual, a distancia. Es decir, permite al estudiante realizar en casa, haciendo uso de herramientas multimedia, lo que se hace en el aula de manera tradicional y lo que frecuentemente se hace en casa (como los deberes), se realiza en el aula, poniendo en práctica los diversos métodos interactivos de trabajo colaborativo. (Wendorff, 2019).

Velásquez (2017), el aula invertida permite desarrollar contenidos previos a la clase y fomenta en el estudiantes la comprensión de los contenidos a través de una aprendizaje activo. El Aula Invertida hace uso de tecnología multimedia con el fin de acceder a los diferentes materiales y herramientas permitiendo el apoyo fuera del aula y poniendo énfasis en la adquisición de las competencias digitales. (Prieto, et al., 2019).

El Aula Invertida es importante para potencializar el desarrollo de las competencias (mejorar el nivel académico); en la parte social busca resolver problemas y así contribuir a la comunidad no solo en el entorno educativo sí no, mejorando los niveles de aprendizaje y el ambiente de estudio utilizando estrategias pedagógicas, que conlleven a los niños a sentirse motivados, realizando actividades que propicien un aprendizaje y entorno creativo, didáctica e interactiva. (Cortes y Riveros, 2019, p. 12).

Al usar el aula invertida en el proceso de enseñanza aprendizaje es importante considerar el enfoque pedagógico que fundamenta el aprendizaje invertido teneindo en cuenta los 4 pilares fundamentales resumidos en el acrónimo "F-L-I-P", según The Flipped Learning Network (2014): ambiente flexible, cultura de aprendizaje, contenido dirigido y facilitador profesional. (Hernández y Tecpan, 2017 y Wendorff, 2019).

El Aula Invertida, lograr personificación individual asegurando la educación de los estudiantes mediante la estructura de necesidades que conlleva inicialmente a pensar de manera desordenada, cuando en realidad es todo lo contrario, es decir la metodología que torna en el aula invertida tiene mayor auge dentro como fuera del aula, Olaizola (2015).Es decir, el aula invertida es visto como un modelo pedagógico acompañado de herramientas digitales, que se enfoca en lograr un aprendizaje activo y efectivo en el estudiante; con relación a los docentes, ellos son los encargado de preparar el material del curso, usando grabaciones de videos existente o grabaciones de ellos mismos explicando la clase, estos materiales son entregados a los estudiantes antes de asistir a clase, esto es ventajoso ya que el estudiante llega al aula con un conocimiento previo del tema permitiendo hacer preguntas, disipar sus dudas y dar apreciaciones, permite centrar al docente en un rol de tutor y guía dando así el protagonismo al estudiante en el proceso de aprendizaje.( Fernández y Godoy, 2017)

El Aula Invertida es un modelo pedagógico no tradicional que, de la mano con las herramientas digitales, busca el aprendizaje efectivo y competente en los estudiantes. Invierte los momentos tradicionales de la relación docente estudiante, donde el docente prepara previamente material de su curso, ya sea grabándose él mismo dictando algún tema de su clase, o recopilando videos ya existentes en la web. Luego, este material se distribuye a los educandos antes de asistir a clases para que ellos puedan verlo en sus hogares, llegando a clases con un conocimiento previo de la materia en cuestión. El momento en el aula se centra en los comentarios, preguntas, dudas y apreciaciones sobre lo observado, teniendo el docente un rol de tutor y guía, mientras que el estudiante es protagonista de su propio aprendizaje. (Acevedo, et al., 2019, Rivas, 2020)

Los denominados "millennials" son nativos digitales, esto obliga a los docentes utilizar estrategias de enseñanza aprendizaje acorde con el contexto, es decir, haciendo uso de metodología 
innovadoras que busquen la atención y motivación del estudiante, logrando hacer uso del desarrollo de las tecnologías de la información y la comunicación basadas en internet poniendo en práctica un aprendizaje mixto o llamado "blended learning" o en español "aprendizaje mezclado". Blasco, Lorenzo y Sarsa (2016). En este sentido, haciendo uso del aula invertida, el docente se convierte en guía, en facilitador del aprendizaje, propiciando el uso de recursos y herramientas necesarias para el desarrollo de las destrezas y habilidades del estudiante. (Salinas, 2004).

El uso del aula invertida permite un aprendizaje invertido o "Flipped Classroom", donde los docentes pones recursos y herramientas de acceso fácil y libre y es el estudiante que debe revisar y usar dichos elementos para conocer las lecciones de curso en cualquier momento. (Bergmann y Sams, 2017 y Vidal, et al., 2016). Asimismo, es importante conocer la estructura del aula invertida, haciendo énfasis en el desarrollo de actividades fuera del aula: a) selección o producción y distribución del material digital; b) actividades para asegurar el visionado y c) diagnóstico de la comprensión del material. Es importante mencionar, que el rol del docente es visto como un productor de contenidos y actividades para ser utilizados por los estudiantes y que el docente pueda evaluar el aprendizaje. (Abio, et al., 2017 y Cedeño, Vigueras 2020).

En la educación superior lograr poner en práctica los procesos cognitivos de nivel superior muchas veces se hace complicado y por ende la dificultad de lograr competencias en los estudiantes, como se conoce en educación superior no se tiene al docente todo el día, la presencia es muy limitado. Es allí que la metodología en la que se toman en cuenta lo tecnológico "Aula invertida permite el uso efectivo ya que se puede trabajar todos los contenidos del curso teniendo en cuenta el enfoque instructivo dentro del aula. Al hacer uso del aula invertida los estudiante tienen al docente disponible todo el tiempo logrando así una retroalimentación de los contenidos de las herramientas proporcionada por el docente antes de la explicación de la clase. (O'Flaherty y Phillips, 2015 y Domínguez et al., 2017).

\section{Método}

La investigación se realizó bajo el enfoque cuantitativo, con el método hipotético deductivo, mediante el planteamiento de hipótesis, la recolección de datos, y el empleo de estadística para obtener los resultados. Así mismo, el diseño fue experimental y el tipo de cuasi experimental, empleándose la U Mann-Whitney para identificar la influencia entre las variables. La muestra seleccionada para el estudio estuvo conformada por estudiantes de una universidad de Lima, integrada por 73 estudiantes de ambos sexo del IV ciclo de la carrera de educación secundaria de la especialidad de idioma extranjero- Inglés. El muestreo fue no probabilístico de tipo intencional y por conveniencia, la muestra fue dividida en dos grupos: Grupo experimental (GE $=36$ estudiantes) y Grupo control ( $\mathrm{GC}=37$ estudiantes). Las variables que se midieron en el estudio fueron aula invertida y aprendizaje autónomo. Las dimensiones de la variable aprendizaje autónomo son: planeación, monitoreo y evaluación. Para medir la variable el aula invertida se utilizaron módulos (recursos y herramientas: videos y grabaciones). Con respecto a la variable aprendizaje autónomo, se elaboró un cuestionario de 20 preguntas, dicho cuestionario pasó por dos etapas: validez que fue hecha por 3 expertos y la confiabilidad lo cual se obtuvo mediante KR20, obteniendo ,864. Con relación al método de análisis de luego se elaboró la base de datos en la hoja de cálculo Excel una para el pretest y otra para el postest.

\section{Resultados.}

En la tabla 1 se aprecia que los resultados del pretest en relación con el aprendizaje autónomo un 
$37,6 \%$ y $41,2 \%$ de los estudiantes del grupo control (GC) y grupo experimental (GE) mantienen un nivel bajo, asimismo, un 57,1\% y 41,5\% presentan un nivel medio, asimismo, el 5,3\% del grupo control y el $17,3 \%$ del grupo experimental se encuentra ubicado en el nivel alto. Por lo que se determina que antes de aplicar el modelo de pedagogía invertido ambos grupos de estudiantes presentan similares condiciones en cuanto al manejo del aprendizaje autónomo.

Con relación a los resultados del postest, es decir, la aplicación de la metodología del aula invertida en los estudiantes del grupo experimental, con las mismas condiciones en el grupo control, un $29,2 \%$ se encuentran en el nivel bajo, el 64,4\% en el nivel medio y un 6,4\% en el nivel alto; sin embargo, en el grupo experimental el $27,9 \%$ de los estudiantes presentan un nivel medio y el $72,1 \%$ lograron puntuar hacia valores altos. Estos porcentajes evidencian cambios relevantes en los estudiantes del grupo experimental debido al incremento considerable de estudiantes que mejoraron su aprendizaje autónomo.

Tabla 1.

Aprendizaje Autónomo en los estudiantes grupo control y experimental según pretest y postest.

\begin{tabular}{|c|c|c|c|c|c|}
\hline & & \multicolumn{4}{|c|}{ Grupo } \\
\hline & & \multicolumn{2}{|c|}{$\begin{array}{c}\text { Control } \\
N=36\end{array}$} & \multicolumn{2}{|c|}{$\begin{array}{c}\text { Experimental } \\
\mathbf{N}=\mathbf{3 7}\end{array}$} \\
\hline & & $\mathbf{F i}$ & $\% \mathrm{Fi}$ & $\mathbf{F i}$ & $\% \mathrm{Fi}$ \\
\hline \multirow[t]{3}{*}{ Pretest } & Bajo & 16 & 37,6 & 15 & 41,2 \\
\hline & Medio & 19 & 57,1 & 17 & 41,5 \\
\hline & Alto & 1 & 5,3 & 5 & 17,3 \\
\hline \multirow[t]{3}{*}{ Postest } & Bajo & 12 & 29,2 & 0 & 0,0 \\
\hline & Medio & 20 & 64,4 & 12 & 27,9 \\
\hline & Alto & 4 & 6,4 & 25 & 72,1 \\
\hline
\end{tabular}

Nota: $\mathrm{n}=73$

Teniendo en cuenta la hipótesis general planteada para el estudio ¿Cuál es la influencia del aula invertida como herramienta para el logro de aprendizaje autónomo en estudiantes universitarios? y de acuerdo al objetivo "Determinar la influencia del aula invertida como herramienta para el logro de aprendizaje autónomo en estudiantes universitarios", en la Tabla 2 se comprobaron las hipótesis propuestas fue necesario establecer qué tipo de prueba será implementada en la investigación. En tal sentido se realizó una prueba de normalidad con la intensión de establecer si la variable dependiente (aprendizaje autónomo) en la población tiene una distribución normal, de ser así se utilizó algunas de las pruebas paramétricas (como es el caso de la prueba t para muestras independientes), de lo contrario se recurrirá a las pruebas no paramétricas (ejemplo la prueba de Mann-Whitney, que es equivalente a la prueba t). 
Tabla 2.

Prueba de normalidad de Shapiro-Wilk: variable aprendizaje autónomo y sus dimensiones, según pretest y postest.

\begin{tabular}{|c|c|c|c|c|}
\hline & \multirow{2}{*}{ Grupo } & \multicolumn{3}{|c|}{ Shapiro-Wilk } \\
\hline & & Estadístico & gl & Sig. \\
\hline \multirow[t]{2}{*}{ Pretest Planeación } & Control & 0,802 & 37 & 0,000 \\
\hline & Experimental & 0715 & 36 & 0,000 \\
\hline \multirow{2}{*}{ Pretest Monitoreo } & Control & 0,873 & 37 & 0,001 \\
\hline & Experimental & 0,822 & 36 & 0,000 \\
\hline \multirow[t]{2}{*}{ Pretest Evaluación } & Control & 0,876 & 36 & 0,000 \\
\hline & Experimental & 0,812 & 37 & 0,000 \\
\hline \multirow[t]{2}{*}{ Pretest } & Control & 0,875 & 37 & 0,534 \\
\hline & Experimental & 0,834 & 37 & 0,001 \\
\hline \multirow[t]{2}{*}{ Postest Planeación } & Control & 0,871 & 36 & 0,000 \\
\hline & Experimental & 0,824 & 36 & 0,000 \\
\hline \multirow[t]{2}{*}{ Postest Monitoreo } & Control & 0,874 & 37 & $\mathbf{0 , 3 1 3}$ \\
\hline & Experimental & 0,834 & 36 & 0,000 \\
\hline \multirow[t]{2}{*}{ Postest Evaluación } & Control & 0,870 & 37 & 0,001 \\
\hline & Experimental & 0,894 & 37 & 0,000 \\
\hline \multirow[t]{2}{*}{ Postest } & Control & 0,872 & 37 & 0,618 \\
\hline & Experimental & 0,859 & 37 & 0,000 \\
\hline
\end{tabular}

Teniendo en cuanta los datos de la prueba de normalidad se plantearon las hipótesis estadísticas. Ho: Las puntuaciones obtenidas en el aprendizaje autónomo tiene una distribución normal.

Hi: Las puntuaciones obtenidas en el aprendizaje autónomo son distintas a la distribución normal.

Se observa en la Tabla 2 la mayoría de los valores de significación resultaron ser menores a 0.05 , a excepción del pretest $) 0,534)$ y postest $(0,618)$ en el grupo control, así como del postest en la dimensión monitoreo $(0,313)$ que son mayores a 0,05 , por lo que las puntuaciones obtenidas en los estudiantes, en particular del GE no se caracterizan por presentar una distribución normal, lo cual conlleva al uso de la prueba Mann-Whitney (U), como estadístico para probar si la variable independiente aula invertida tiene influencia en el aprendizaje autónomo en los estudiantes.

\section{Hipótesis general}

Ho: El aula invertida tiene influencia como herramienta para el logro de aprendizaje autónomo en estudiantes universitarios.

Hi: El aula invertida no tiene influencia como herramienta para el logro de aprendizaje autónomo en estudiantes universitarios. 
Tabla 3.

Rangos según test

\begin{tabular}{llccc}
\hline \multicolumn{5}{c}{ Rangos } \\
\hline & Grupo & N & Rango promedio & Suma de rangos \\
\hline Puntaje en el pretest & Control & 36 & 33,24 & 1023,40 \\
& Experimental & 37 & 37,12 & 1563,30 \\
& Total & 73 & & \\
\hline Puntaje en el postest & Control & 36 & 1989 & 667,00 \\
& Experimental & 37 & 49,96 & 1853,20 \\
& Total & 73 & & \\
\hline
\end{tabular}

Se aprecia que en el pretest los rangos promedios de los estudiantes correspondiente a los grupos control y experimental presentaban similitud, no obstante, una vez que los sujetos del GE recibieron los talleres de la metodología del aula invertida se obtuvieron cambios significativos al comparar los promedios de este grupo antes y después se observó que hubo una diferencia de 12,84 puntos, lo que indica que la metodología del aula invertida tiene influencia en el aprendizaje autónomo. Tabla 4.

Nivel de comprobación y significación estadística entre los test para el aprendizaje autónomo

\begin{tabular}{lcc}
\hline & Estadísticos de contraste $^{\mathbf{a}}$ \\
\hline U de Mann-Whitney & Pretest & Postest \\
W de Wilcoxon & 492,200 & 81,100 \\
$\mathrm{Z}$ & 1456,120 & 776,100 \\
Sig. asintót. (bilateral) & $-0,824$ & $-5,434$ \\
\hline
\end{tabular}

a. Variable de agrupación: Grupo

Según los datos señalados en la Tabla 4 se tiene que en el pretest el valor asociados al estadístico U de Mann-Whitney resultó ser 0,428 que es mayor al $p$ 0,05 lo que permite concluir que antes de la aplicación del taller aula invertida los dos grupos de estudios presentaban similares condiciones con relación al aprendizaje autónomo, por lo que se acepta la hipótesis nula. Asimismo, el valor $Z$ calculado $(-0,824)$ es menor al valor tipificado de $-1,96$ lo que confirma la aceptación de la hipótesis nula.

Con referencia al postest de los resultados se aprecia los estadísticos de los grupos de estudio, siendo el nivel de significancia ( $\mathrm{p}=0.000$ ) menor que 0.05 y $\mathrm{Z}$ igual a -5.434 menor que -1.96 lo que permite rechazar la hipótesis nula, es decir, la metodología del aula invertida tiene influencia en el aprendizaje autónomo.

\section{Discusión}

La finalidad del presente estudio fue Determinar la influencia del aula invertida como herramienta para el logro de aprendizaje autónomo en estudiantes universitarios teniendo como resultado tanto del pretes como del post test en relación al aprendizaje autónomo de los estudiantes un 37,6\% y $41,2 \%$ del grupo control (GC) y grupo experimental (GE) mantienen un nivel bajo, asimismo, un $57,1 \%$ y $41,5 \%$ presentan un nivel medio, además, el 5,3\% del grupo control y el 17,3\% del grupo experimental se encuentra ubicado en el nivel alto. Los que indica que ambos grupos presentan similares condiciones en cuanto al manejo del aprendizaje autónomo. Dichos resultados coinciden con los de Carignano (2016) el aula invertida como el impacto de la tecnología como herramienta 
para superar las deficiencias en los resultados académicos. En las universidades del Perú existen docentes que usan el método tradicional en lo cual hace que la realidad de la educación peruana tenga la necesidad de replantear nuevas metodologías como el aula invertida para que el estudiante logre desarrollar los proceso cognitivos superiores y lograr un aprendizaje autónomo. En la metodología aula invertida es necesario la participación del docente facilitador de los recursos y herramientas y del estudiante, que es el protagonista activo en el proceso de enseñanza aprendizaje. La metodología del aula invertida tiene como fin abordar el problema de cambio de una educación tradicional logrando usar recursos digitales realizados por los docentes y ser entregados a los estudiantes antes de la clase, el estudiante al usar dichos recurso tecnológicos disponibles logra no solo ser responsable de su propio aprendizaje, sino que se considere una persona con valores en la que el afecto y las emociones sean características únicas en él.

Con relación a los resultados del postest, es decir, la aplicación de la metodología del aula invertida en los estudiantes del grupo experimental, con las mismas condiciones en el grupo control, un 29,2\% se encuentran en el nivel bajo, el 64,4\% en el nivel medio y un 6,4\% en el nivel alto; sin embargo, en el grupo experimental el $27,9 \%$ de los estudiantes presentan un nivel medio y el $72,1 \%$ lograron puntuar hacia valores altos, lo que se evidencian cambios relevantes en los estudiantes del grupo experimental debido al incremento considerable de estudiantes que mejoraron su aprendizaje autónomo. Dichos resultados coinciden con Blasco, Lorenzo y Sarsa (2016) Concluyó que el modelo de Flipped Classroom logra influir de manera positiva en las competencias transversales, es decir el aula invertida el planteamiento se hace más exigente desde la planificación como la organización en la medida del abordaje de los materiales y recursos útiles para el proceso enseñanza aprendizaje. En este sentido tiene mucho que ver el involucramiento también de la institución, la universidad, es decir, desde la reestructuración documental, organización, distribución de los ambientes, sobre todo capacitación y actualización de los docentes en temas de tecnología y materiales digitales.

Al realizar la comparación de los rangos correspondiente a los grupos control y experimental presentaban similitud, no obstante, una vez que los sujetos del GE recibieron los talleres de la metodología del aula invertida se obtuvieron cambios significativos al comparar los promedios de este grupo antes y después se observó que hubo una diferencia de 12,84 puntos, lo que indica que la metodología del aula invertida tiene influencia en el aprendizaje autónomo. Este resultado difiere de lo Zacarías (2016) se utilizaron TIC como recursos para apoyar en el aprendizaje de los estudiantes y se identificaron los factores que influyen en la falta de difusión de la metodología Flipped Classroom.

El uso del aula invertida permite a los docentes pones recursos y herramientas de acceso fácil y libre y es el estudiante que debe revisar y usar dichos elementos para conocer las lecciones de curso en cualquier momento. Los datos obtenidos en el pretest el valor asociados al estadístico $U$ de Mann-Whitney resultó ser 0,428 que es mayor al p 0,05 lo que determina que antes de la aplicación del taller aula invertida los dos grupos de estudios presentaban similares condiciones con relación al aprendizaje autónomo, por lo que se acepta la hipótesis nula. Asimismo, el valor Z calculado ($0,824)$ es menor al valor tipificado de $-1,96$ lo que confirma la aceptación de la hipótesis nula. Estos resultado presentan similitud con Zacarías (2016) concluye que sí hubo efectos positivos en cuanto al aprendizaje de los estudiantes y que además es necesario adoptar nuevas metodologías para la enseñanza aprendizaje, como también el uso de TIC como recursos para apoyar en el aprendizaje de los estudiantes y se identificaron los factores que influyen en la falta de difusión de la metodología Flipped Classroom. Con referencia al postest de los resultados se aprecia los estadísticos de los grupos de estudio, siendo el nivel de significancia $(\mathrm{p}=0.000)$ menor que 0.05 y $\mathrm{Z}$ igual a -5.434 
menor que -1.96 lo que permite rechazar la hipótesis nula, es decir, la metodología del aula invertida tiene influencia en el aprendizaje autónomo. Finalmente los resultados de Carignano (2016) determinó que el aula invertida se convirtió en un ambiente de aprendizaje activo, donde el estudiante está involucrado directamente en el aprendizaje, lo que confirma el resultado de la investigación. Se recomienda que los docentes implementen y use el Aula Invertida en educación superior, en los diferentes cursos, teniendo conciencia que las metodologías están en constante evolución según el contexto, asimismo, debe haber involucramiento entre todos los actores del proceso educativo en beneficio de la educación.

\section{Conclusiones}

Conclusión 1: Determinar la influencia del aula invertida como herramienta para el logro de aprendizaje autónomo en estudiantes universitarios se determinó con la búsqueda de metodología de aula invertida lo que permitió afianzar que la metodología de aula invertida es de gran importancia, debido que permite que el estudiante logre desarrollar un aprendizaje autónomo esto se corrobora con los resultados en el pretest el valor asociados al estadístico U de Mann-Whitney resultó ser 0,428 que es mayor al $p$ 0,05 lo que permite concluir que antes de la aplicación del taller aula invertida los dos grupos de estudios presentaban similares condiciones con relación al aprendizaje autónomo, por lo que se acepta la hipótesis nula. Asimismo, el valor $Z$ calculado $(-0,824)$ es menor al valor tipificado de $-1,96$ lo que confirma la aceptación de la hipótesis nula. Con referencia al postest de los resultados se aprecia los estadísticos de los grupos de estudio, siendo el nivel de significancia $(\mathrm{p}=0.000)$ menor que 0.05 y $\mathrm{Z}$ igual a -5.434 menor que -1.96 lo que permite rechazar la hipótesis nula, es decir, el aula invertida, permite al estudiante aprender de manera autónoma incorporando las TIC como elemento innovador en la trasformación de la adquisición de los nuevos aprendizajes de manera responsable.

Conclusión 2: el aula invertida como metodología permite el aprovechamiento de los diferentes recursos como son las TIC, el aprendizaje colaborativo, pero sobre todo la parte emocional y afectiva del ser humano, al aplicar el aula invertida en el estudio se determinó que antes de aplicar el modelo de pedagogía invertido ambos grupos de estudiantes presentan similares condiciones en cuanto al manejo del aprendizaje autónomo, a diferencia del grupo experimental se obtuvo un incremento considerable de estudiantes que mejoraron su aprendizaje autónomo.

Conclusión 3: el fin del proceso enseñanza aprendizaje es que el estudiante logre las competencias pero sobre todo desarrolle un aprendizaje autónomo y para lograr eso es importante el uso de metodologías activas como el aula invertida "Flipped Classroom", pero para lograr eso el docente debe dejar de ser reacio al cambio y hacer uso de las metodologías activas existente que le permitirán al docente ser el guía, el facilitador de recurso y herramientas y por parte el estudiante lograr aprendizajes, estilos, ritmos y toma de conciencia en su educación. 


\section{Referencias}

Abio, G., Alcañiz, M. Gómez, M. Rubert, G. Serrano, M. Stoyanova, A. y Vilalta, M. (2017). El aula invertida y el aprendizaje en equipo dos metodologías para estimular al alumnado repetidor. Revista d'Innovació Docent Universitària 9, pp. 1-15 http://revistes.ub.edu/index.php/RIDU

Acevedo, A., Prada, D. Ramírez, J. Chia, M. y Roman, J. (2019). Aula Invertida para la mejora de la cultura financiera del estudiantado Santandereano: Caso Concurso Bolsa Millonaria (Colombia). Revista espacios Vol. 40 (44) 8. https://bit.ly/3a6vbVy

Archbold, F. A., Nuñez, L. J. \& Padilla, L. F. (2019). Aula invertida: análisis de una experiencia disruptiva en la práctica de enseñanza y aprendizaje desde la mirada docente. Recuperado de: http://hdl.handle.net/10554/46606.Arráez, Lorenzo, A., Gómez y Lorenzo, G., 2018

Arráez, G., Lorenzo, A., Gómez, M., \& Lorenzo, G. (2018). La clase invertida en la educación superior: percepciones del alumnado. Revista INFAD de Psicología. International Journal of Developmental and Educational Psychology., 2(1), 155-162. doi:https://doi.org/10.17060/ijodaep.2018.n1.v2.1197

Bergmann, J. y Sams, A. (2014). Dale la vuelta a tu clase. Lleva tu clase a cada estudiante, en cualquier momento y cualquier lugar. España: Ediciones SM 2014.

Bergmann, J., \& Sams, A. (2017). Flipped Learning: Maximizing Face Time. Td.org. Retrieved 6 July 2017, from https://www.td.org/Publications/Magazines/TD/TDArchive/2014/02/Flipped-Learning-Maximizing-Face-Time

Blasco, A., Lorenzo, J. y Sarsa, J. (2016). La clase invertida y el uso de videos de software educativo en la formación inicial del profesorado. Estudio cualitativo. @ tic. revista $\begin{array}{lllll}\text { d'innovació } & \text { educativa., } & 17, & 12-20 . & \text { ISSN: }\end{array}$ https://roderic.uv.es/handle/10550/57153

Carignano, C. (2016). Implementación de clase invertida en una escuela de una universidad de Lima Metropolitana (Tesis de maestría). Del repositorio de la Pontificia Universidad Católica del Perú. Recuperado de: https://bit.ly/2MX5jme

Cedeño, M., \& Vigueras, J. (2020). Aula invertida una estrategia motivadora de enseñanza para estudiantes de educación general básica. Dominio de las Ciencias, 6(3), 878-897. doi:http://dx.doi.org/10.23857/dc.v6i3.1323

Cortés, L. y Riveros, C. (2019). El aula invertida como herramienta tic's para el aprendizaje de la asignatura de sistemas en el grado primero $\left(1^{a}\right)$ en el colegio san Nicolás del espinal Tolima. p.21-24. Recuperado de: https://bit.ly/3aUE43H

Domínguez, L. C., Sierra, D., Pepín, J. J. Moros, G. y Villarraga, A. (2017). Effect of the Extended Inverted Classroom on clinical simulation for the resuscitation of trauma patients: Pilot study of student perceptions of learning Colombian. Journal of Anesthesiology, Volume 45, 2,4-11. https://bit.ly/2MMYIuS

Fernández, M. G. y Godoy, M. V. (2017). Aula Invertida para la inclusión de Recursos Educativos Abiertos Enseñanza de Tecnología Aplicada al Arte. https://bit.ly/3aVARku

Flipped Learning Network [FLN]. (2014). Definition of Flipped Learning. Retrieved from http://flippedlearning.org/domain/46

Hernández, C. y Tecpan, S. (2017). Aula invertida mediada por el uso de plataformas virtuales: un estudio de caso en la formación de profesores de física. Estudios Pedagógicos, XLIII (3), 193-204. ISSN: 0716-050X. https://www.redalyc.org/articulo.oa?id=1735/173554750011

Kong, S. C. (2014). Developing information literacy and critical thinking skills through domain knowledge learning in digital classrooms: An experience of practicing flipped classroom strategy. Computers \& Education, 78, 160-173.

Limón, M., Cantera, E. y Salinas, L. (2017). Aprendizaje invertido: una propuesta de 
enseñanzaaprendizaje en una clase de cálculo diferencial. Revista de Pedagogía Critica.1 1,10-15. https://bit.ly/3paI6KD.

Madrid, E. M., Angulo, J., Prieto, M. Fernández, M, T y Olivares, K. M. (2018). Implementación de aula invertida en un curso propedéutico de habilidad matemática en bachillerato. Revista apertura Volumen 10, número 1, pp. 24-39 http://dx.doi.org/10.32870/Ap.v10n1.1149

McLaughlin, J. E., Roth, M. T., Glatt, D. M., Gharkholonarehe, N., Davidson, C. A., Griffin, L. M., Mumper, R. J. (2014). The flipped classroom: A course redesign to foster learning and engagementin a health professions school. Academic Medicine: Journal of the Association of American Medical Colleges, 89(2), 236-243. doi:10.1097/ACM.0000000000000086

Merla, A. Yáñez, C. (2016). El aula invertida como estrategia para la mejora del rendimiento académico. Revista mexicana de bachillerato a distancia, 8(16), 74. Recuperado de: https://bit.ly/3jBsZbX

O'Flaherty, J. y Phillips, C. (2015). The use of flipped classrooms in higher education: A scoping review. The Internet and Higher Education, 25, 85-95.

Olaizola, A. (2015). La Clase Invertida: Usar las TIC para "dar vuelta" a la clase. Recuperado de: https://bit.ly/3qchCcR

Prieto, A., Barbarroja, J., Lara, I. Díaz, D., Pérez, A., Monserrat, J., Corell, A., \& Álvarez de Mon, M. (2019). Aula invertida en enseñanzas sanitarias: recomendaciones para su puesta en práctica. FEM: Revista de la Fundación Educación Médica, 22(6), 253-262. Recuperado de https://bit.ly/3d1Bqfl.

Rivas, V. R. (2020). El aula invertida una estrategia educativa en el modelo híbrido. Revista Guatemalteca de Educación Superior, 3(2), 136-145. DOI: https://doi.org/10.46954/revistages.v3i2.39

Rodríguez, M. y García, R. (2016). El aula invertida (flipped classroom) en educación primaria: un estudio de caso. Universidad de Sevilla, 2016. Recuperado de:141 https://bit.ly/3a7f22c

Salinas, J. (2004). Innovación docente y uso de las TIC en la enseñanza universitaria. Revista Universidad y Sociedad del Conocimiento (RUSC). Vol. 1 - Nº 1 , noviembre de 2004, pp. 1-16, Universitat Oberta de Catalunya, España. DOI: http://dx.doi.org/10.7238/rusc.v1i1.228

Sierra, M. A. y Mosquera, F. M. (2020). El aula invertida como estrategia pedagógica para mejorar el aprendizaje en estudiantes de educación presencial. Tesis Universidad Nacional Abierta y a Distancia -UNAD. Escuela Ciencias de la Educación (ECEDU). Recuperado de: https://bit.ly/3tOqo39

Velásquez, R. (2017). ¿Es efectiva realmente el aula invertida o flipped classroom? Recuperado de:https://www.oei.es/historico/divulgacioncientifica/?Es-efectiva-realmente-el-aulainvertida-o-flipped-classroom.

Vidal, M., Rivera, N., Nolla N., Morales, I. del R., \& Vialart, M. N.. (2016). Aula invertida, nueva estrategia didáctica. Educación Médica Superior, 30(3), 678-688. Recuperado en 10 de febrero de 2021, de https://bit.ly/2Z53c2h

Wendorff, C. A. (2019). Aula invertida para el aprendizaje de dominio en los estudiantes del curso de metodología de la investigación de una universidad privada de Lima,Tesis maestría Universidad San Ignacio de Loyola Lima - Perú: Recuperado de: https://bit.ly/2Z6uHbK

Zacarías, V (2016). Relación entre la metodología Flipped Classroom y el aprendizaje de alumnos en la Universidad Continental mediante el uso de TIC. Recuperado de://recursos.portaleducoas.org/sites/default/files/VE16.648.pdf 